\title{
Ferromagnetism in II-VI Based Semiconductor Structures
}

\author{
J. Cibert ${ }^{a}$, D. Ferrand ${ }^{a}$, S. Tatarenko $^{a}$, A. Wasiela $^{a}$, \\ P. KOSSACKI ${ }^{b}$ AND T. DIETL ${ }^{c}$ \\ ${ }^{a}$ Laboratoiré de Spectrométrie Physique, CNRS-Université J. Fourier, Grenoble \\ BP87, 38402 Saint Martin d'Hècres cedex, France \\ ${ }^{b}$ Institute of Experimental Physics, Warsaw University \\ Hoża 69, 00-681 Warszawa, Poland \\ ${ }^{c}$ Institute of Physics, Polish Academy of Science \\ Al. Lotników 32/46, 02-668 Warszawa, Poland
}

\begin{abstract}
The experimental results on carrier-induced ferromagnetic interaction in II-VI diluted magnetic semiconductors are shortly reviewed and analyzed in the light of the mean field approximation. We particularly take the point of view of the experimentalist to emphasize (i) points which are easily understood within this simple model (as the role of the detailed structure of the valence band) and hence should be incorporated into more sophisticated models, and (ii) points which are not taken into account in the mean field model (e.g., disorder effects) and hence call for more elaborate descriptions. We particularly discuss the case of a low carrier density, and show that the situation appears as experimentally very different in the highly disordered $3 \mathrm{D}$ layers and in the modulation doped $2 \mathrm{D}$ quantum wells.
\end{abstract}

PACS numbers: 72.80.Ey, 75.50.Pp, 75.30.Hx, 75.50.Dd

\section{Introduction}

There is much work done currently on diluted magnetic semiconductors where carriers (generally holes in the valence band) induce a ferromagnetic interaction between localized spins (generally Mn impurities). Examples include PbTe-based bulk samples [1], and more recently semiconductor compounds with the zinc-blend structure. Among them III-V semiconductors (GaMnAs and InMnAs) [2, 3] exhibit quite high critical temperatures. As Mn acts both as 
an acceptor and carries the spin in these compounds, their theoretical analysis has proven to be difficult. However they are very attractive for applications, and the control of the ferromagnetic interaction through an electrostatic gate has been demonstrated [4]. In the II-VI semiconductors (CdMnTe [5], ZnMnTe [6], BeMnTe [7]), the Mn is isoelectronic and the $p$-type doping is controlled independently by adding nitrogen acceptors. Thus, in spite of the low critical temperatures which have been observed, these compounds are very attractive in order to gain a better understanding of the mechanisms: They can be elaborated with a great variety of carrier density and spin content, in various configurations (3D thick layers and $2 \mathrm{D}$ quantum wells), and the material parameters (in particular the spin carrier coupling) are accurately known from previous studies of the undoped materials. Finally, we should mention that the quest for materials with higher critical temperatures is currently pursued either theoretically [8-10] or experimentally [11-13].

In the following we shall give a short overview of the experimental results in ZnMnTe 3D layers and in CdMnTe 2D quantum wells, in the light of the mean field model [14] which surprisingly well accounts for the observed critical temperatures. This model will particularly be used to put into evidence some experimental observations which claim for more sophisticated models, but also to emphasise the role of the detailed structure of the valence band - a point which certainly has to be incorporated into the more sophisticated models [15-17].

\section{The mean field model}

The coupling between a spin $\boldsymbol{S}_{i}$ localized at $\boldsymbol{R}_{i}$ and a hole of spin $\boldsymbol{s}$ and position $r$ is described by the usual local Hamiltonian

$$
-\beta \boldsymbol{S}_{i} \cdot s \delta\left(\boldsymbol{r}-\boldsymbol{R}_{i}\right)
$$

This Hamiltonian has been used for a long time to describe the so-called giant magneto-spectroscopic effects, such as the giant Zeeman effect. The valence-band splitting (and also the conduction band splitting and the exciton splitting), calculated by a mean-field and virtual crystal approximation [18], is proportional to the magnetization of the Mn system. Hence the spin-carrier parameter $(\beta)$ is well known from previous spectroscopic studies on undoped materials and well documented in the literature [19]. In addition, once $\beta$ is determined, spectroscopic studies provide a very sensitive way of measuring the Mn magnetization in a sample. And finally, in the case of a doped sample, the carrier polarization can be written as

$$
\left\langle s_{z}\right\rangle=\beta \tilde{\chi}_{\mathrm{h}} M / g \mu_{\mathrm{B}},
$$

where $M$ is the $M n$ magnetization and $\tilde{\chi}_{\mathrm{h}}$ is the spin susceptibility of the carrier (omitting $g \mu_{\mathrm{B}}$ factors), which has to be calculated applying the Fermi-Dirac statistics on the holes in the complex valence band. In the presence of an applied field, the direct effect of the field on the carriers can be neglected for relevant $\mathrm{Mn}$ 
contents. Quite symmetrically, we can perform a mean field approximation on the carrier spin in Eq. (1), in order to calculate its effect on a given Mn spin. One obtains

$$
M / g_{\mathrm{Mn}} \mu_{\mathrm{B}}=-x_{\mathrm{eff}} N_{0}\left\langle S_{z}\right\rangle=\tilde{\chi}_{\mathrm{Mn}}\left\{g_{\mathrm{Mn}} \mu_{\mathrm{B}} H-\beta\left\langle s_{z}\right\rangle\right\}
$$

where the direct effect of the applied field has to be considered. The susceptibility of the Mn system takes into account the effect of Mn-Mn superexchange interaction, which is well known in these materials [19]. To that purpose, in the relevant range of fields and temperatures, one writes in a phenomenological approach

$$
\tilde{\chi}_{\mathrm{Mn}}=\frac{\tilde{C}_{0} x_{\mathrm{eff}}}{T+T_{\mathrm{AF}}},
$$

where the effective density of free spins $x_{\mathrm{eff}}$ takes into account the blocking of nearest neighbour pairs, and $T_{\mathrm{AF}} \approx$ a few $\mathrm{K}$ represents the interaction between more distant spins. In Eq. (4) too, we have omitted $g \mu_{\mathrm{B}}$ factors, so that the reduced Curie constant is $\tilde{C}_{0}=S(S+1) N_{0} / 3 k_{\mathrm{B}}$, where the Mn spin $S=5 / 2$ and $N_{0}$ is the density of cation sites.

Once Eq. (2) has been made explicit, Eqs. (2) to (4) are easily combined to obtain a Curie-Weiss behaviour of the Mn system in the presence of the carriers [14], i.e.,

$$
M=g \mu_{\mathrm{B}} \frac{\tilde{\chi}_{\mathrm{Mn}}}{1-\tilde{\chi}_{\mathrm{Mn}} \tilde{\chi}_{\mathrm{h}} \beta^{2}} H=g \mu_{\mathrm{B}} \frac{\tilde{C}_{0} x_{\mathrm{eff}}}{T-T_{\mathrm{CW}}} H
$$

with

$$
T_{\mathrm{CW}}=T_{\mathrm{F}}-T_{\mathrm{AF}} \quad \text { and } \quad T_{\mathrm{F}}=x_{\mathrm{eff}} \tilde{C}_{0} \beta^{2} \tilde{\chi}_{\mathrm{h}} .
$$

In the case of a $3 \mathrm{D}$ system with spins $1 / 2$ fully free to rotate, as would be the case with electrons in the conduction band, the calculated Curie-Weiss temperature simply writes

$$
T_{\mathrm{CW}}=\frac{1}{4} \tilde{C}_{0} x_{\mathrm{eff}} \beta^{2} \rho_{3 \mathrm{D}}\left(E_{\mathrm{F}}\right)-T_{\mathrm{AF}}
$$

with $\rho_{3 \mathrm{D}}\left(E_{\mathrm{F}}\right)$ - the density of states at the Fermi level, while in a quantum well, one has

$$
T_{\mathrm{CW}}=\frac{1}{4} \tilde{C}_{0} x_{\mathrm{eff}} \beta^{2} \rho_{2 \mathrm{D}}\left(E_{\mathrm{F}}\right) / L-T_{\mathrm{AF}},
$$

where $L$ describes the extension of the electron envelope function and is of the order of the quantum well width. However, Eq. (6) evidences the fact that, within this model, the Pauli susceptibility of the hole gas (hence the detailed structure of the valence band) is an important parameter. The $2 \mathrm{D}$ case and the $3 \mathrm{D}$ case have to be considered separately. 


\section{The 3D case: thick layers}

The effect of the spin-orbit coupling on the valence band, in the vicinity of the Brillouin zone centre, is not only to split the band into a doublet and the relevant quadruplet (heavy holes and light holes), but also to align the spin quantification axis on the direction of the $\boldsymbol{k}$-vector. As a result, the spin susceptibility is reduced, since holes propagating along the directions perpendicular to a $z$-axis have no spin component in the $z$ direction, and this leads to a reduction of the spin susceptibility. The complete expression of the longitudinal component of the static spin susceptibility is

$$
\tilde{\chi}_{\mathrm{h}}=2 \sum_{i, j} \sum_{\boldsymbol{k}} \frac{\left|\left\langle i, k\left|s_{z}\right| j, k\right\rangle\right|^{2}}{E_{j, k}-E_{i, k}} f\left(E_{i, \boldsymbol{k}}\right)\left[1-f\left(E_{j, \boldsymbol{k}}\right)\right]
$$

where $|i, k\rangle$ are the periodic part of the Bloch functions, $E_{i, k}=\hbar^{2} k^{2} / 2 m_{\mathrm{hh}}^{*}$ or $\hbar^{2} k^{2} / 2 m_{\mathrm{lh}}^{*}$, and $f\left(E_{i, k}\right)$ is the Fermi-Dirac distribution function for the corresponding hole subbands. Note that in Eq. (8) one has contributions with $i=j$ where both states are either heavy holes or light holes (lying at the Fermi level), and cross terms with $i \neq j$, involving one occupied heavy-hole state and one empty light-hole state. The result that is obtained after a straightforward calculation for the degenerate hole gas assumes the form [6]

$$
\tilde{\chi}_{\mathrm{h}}=\frac{1}{4} A_{\mathrm{F}} \rho\left(E_{\mathrm{F}}\right)\left[\frac{1}{3}+\frac{8}{9} \frac{m_{\mathrm{hh}}^{* 3 / 2} m_{\mathrm{lh}}^{*}-m_{\mathrm{lh}}^{* 3 / 2} m_{\mathrm{hh}}^{*}}{\left(m_{\mathrm{hh}}^{*}-m_{\mathrm{lh}}^{*}\right)\left(m_{\mathrm{hh}}^{* 3 / 2}+m_{\mathrm{lh}}^{* 3 / 2}\right)}\right],
$$

where the Fermi liquid parameter $A_{\mathrm{F}}$ takes the effect of the hole-hole interaction, and

$$
\rho\left(E_{\mathrm{F}}\right)=\left(m_{\mathrm{hh}}^{* 3 / 2}+m_{\mathrm{lh}}^{* 3 / 2}\right)^{2 / 3}\left(3 \pi^{2} \rho\right)^{1 / 3} / \pi^{2} \hbar^{2} .
$$

Layers with various $\mathrm{Mn}$ contents and hole densities have been studied by magnetic measurements and transport (magnetoconductivity and Hall effect). The main results can be summarized as follows [6]:

(i) A ferromagnetic interaction is induced by the presence of the holes, as evidenced by the susceptibility exhibiting a positive Curie-Weiss temperature which well agrees with Eqs. (5, 6, 9, 10) (Fig. 1);

(ii) At temperatures below $T_{\mathrm{CW}}$ one observes remanence in the magnetization cycles.

(iii) The magnetic properties and the transport characteristics strongly interact: A large negative magnetoresistance, and spin-dependent ("extraordinary" or "anomalous") Hall effect, are observed.

A ferromagnetic interaction has been reported also in $\mathrm{Be}_{1-x} \mathrm{Mn}_{x} \mathrm{Te}$ in the presence of a hole gas [7]. It would be interesting to plot the results on this new material into Fig. 1. However, the determination of the spin-carrier coupling is more difficult in this case since BeTe is an indirect semiconductor. 


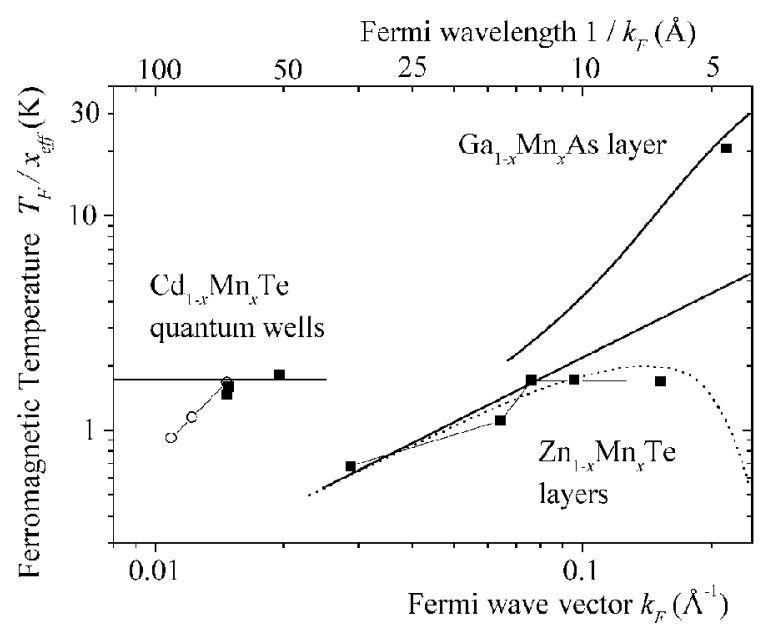

Fig. 1. Results of the critical temperatures measured for $2 \mathrm{D}$ and $3 \mathrm{D}$ samples with different $\mathrm{Mn}$ contents and hole densities. The horizontal scale displays the Fermi wave vector. The vertical scale is $T_{\mathrm{F}}$ as defined in Eq. (6), divided by the effective density of spins $x_{\text {eff }}$. Solid lines are the results of calculations in the mean field model. The dotted line takes into account the RKKY interaction (see Ref. [6]).

It is particularly interesting to compare the results obtained on two different samples with different hole densities and Mn contents.

The transport and magnetic properties of a sample with a high-hole density $\left(p=1.2 \times 10^{20} \mathrm{~cm}^{-3}\right)$ and a low Mn content $(x=0.019)$ are summarized in Fig. 2. The conductivity exhibits a metallic behaviour down to low temperatures. Actually, if a magnetic field high enough is applied to the sample, the measured conductivity is almost identical to that of a ZnTe sample with the same hole density. Without applied field, the low temperature conductivity is smaller [6], but it remains finite down to very low temperatures [20]. Hence it is the presence of magnetic ions which affects the conductivity, but the hole density in this sample ( $p=1.2 \times 10^{20} \mathrm{~cm}^{-3}$ ) is larger than the Mott critical density even if the Mn magnetization is not blocked by a large applied field.

The Hall effect measured on this sample can be decomposed into two terms: A normal Hall effect, linear in field and independent of the temperature, from which we deduced the quoted hole density, and a spin-dependent Hall effect, which is reasonably proportional to the magnetization in the whole field and temperature range (see Fig. 2c which demonstrates that a single scale factor allows us to match the spin dependent Hall effect onto the magnetization).

In addition, Fig. $2 \mathrm{~b}$ displays the magnetization deduced from the spin-dependent Hall effect, and that calculated in the mean field model, on both sides of the critical temperature (keeping the same factor as in Fig. 2c). The only unknown parameter $x_{\mathrm{eff}}$, was deduced from the slope of the dependence of $\chi^{-1}$ on tem- 
(a) $10 \quad 5 \quad$ Temperature (K)

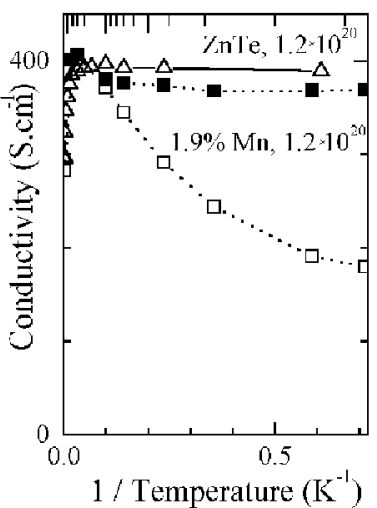

(b)

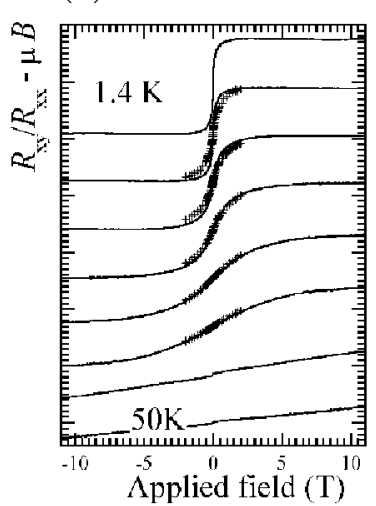

(c)

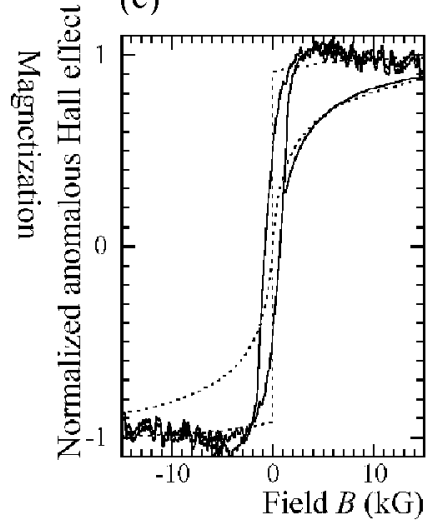

Fig. 2. Transport and magnetic properties of a sample with a high-hole density $\left(p=1.2 \times 10^{20} \mathrm{~cm}^{-3}\right)$ and a low Mn content $(x=0.019)$; (a) conductivity with (closed squares) and without (open squares) a magnetic field (11 T) applied perpendicular to the layers, compared to the conductivity of a ZnTe sample with the same hole density (triangles); (b) comparison of the spin-dependent Hall effect (solid lines) and the magnetization (crosses) at different temperatures $(1.4 \mathrm{~K}, 1.7 \mathrm{~K}, 2.8 \mathrm{~K}, 4.2 \mathrm{~K}, 7 \mathrm{~K}, 10 \mathrm{~K}, 30 \mathrm{~K}$, $50 \mathrm{~K}$ ); the field is applied perpendicular or parallel to the layer, respectively; (c) spin dependent part of the Hall effect (solid lines) compared to the prediction of the mean field model (dotted lines) above $(1.7 \mathrm{~K})$ and below $(0.1 \mathrm{~K})$ the critical temperature.

perature (see Eq. (5)), and a good fit is obtained to the magnetization directly measured above $1.5 \mathrm{~K}$. The comparison in Fig. $2 \mathrm{~b}$ demonstrates the validity of our assumption of a uniform behaviour of both the Mn system and the hole gas. It points also to an almost saturated magnetization of the Mn spins in the ordered phase in this heavily doped sample.

Figure 3 shows the results obtained on a sample with a lower hole density $\left(p=3 \times 10^{19} \mathrm{~cm}^{-3}\right)$ and a higher Mn content $(x=0.038)$.

An insulating behaviour of the conductivity is observed in the absence of an applied field (Fig. 3a). This is due do the presence of Mn, since ZnTe samples with an even lower hole density are metallic [21]. The metallic behaviour appears to be restored by applying a magnetic field, confirming the fact that the metal-insulator transition is induced by the presence of the magnetic impurities.

A transition occurs anyway, with remanence observed in the magnetization cycles measured in the low-temperature phase [6].

Due to the strong increase in the resistivity, by several orders of magnitude (which gives rise to a giant magnetoresistance of the same order at low temperature), we could not make a complete comparison of the spin dependent Hall effect and of the magnetization, as for the previous sample. Figure $3 \mathrm{~b}$ shows that the spin-dependent Hall effect is proportional to the Mn magnetization only at low 
(a) 'l'emperature $(\mathrm{K})$

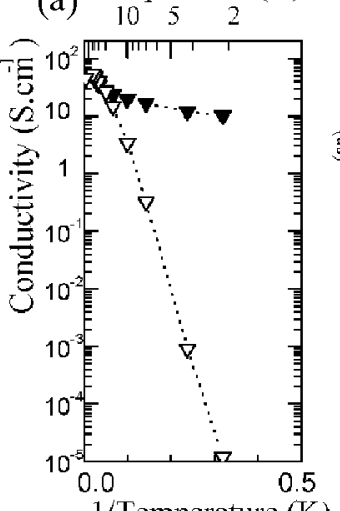

(b)

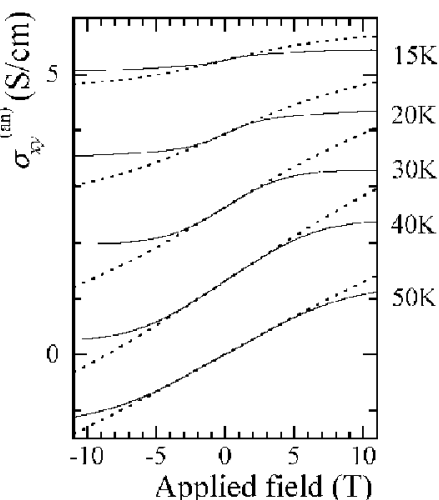

(c)

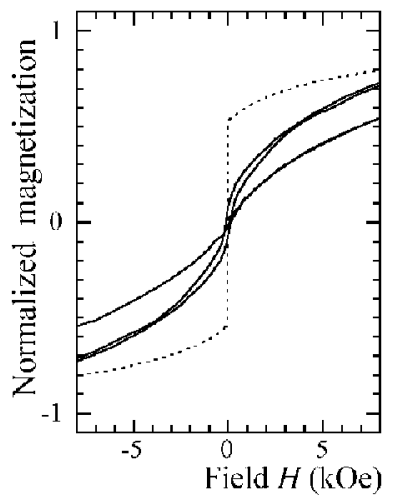

Fig. 3. Transport and magnetic properties of a sample with an intermediate hole density $\left(p=3 \times 10^{19} \mathrm{~cm}^{-3}\right)$ and a higher Mn content $(x=0.038)$ ); (a) conductivity with (closed triangles)) and without (open triangles) a magnetic field (11 T) applied perpendicular to the layers; (b) comparison of the spin-dependent Hall effect (solid lines) and the magnetization (actually Brillouin functions with parameters deduced from the fit of the measured magnetization, dotted lines); (c) magnetization (solid lines) compared to the prediction of the mean field model at $1.7 \mathrm{~K}$ (dotted line).

field, and then abruptly saturates. A detailed interpretation of the spin-dependent Hall effect remains to be performed. However, this observation suggests that the mobile holes are fully polarized at the corresponding field. The most intriguing point is shown in Fig. 3c: It appears that the spontaneous magnetization in the ordered phase is smaller than expected from the uniform model, which suggests that only a part of the Mn spins is involved in the ordered phase, or that a more complex ordered phase is established.

\section{The $2 \mathrm{D}$ case: quantum wells}

The realization of modulation doped $\mathrm{CdMnTe}-\mathrm{CdZnMgTe}$ quantum wells offers the opportunity to study a very different configuration. First, the acceptors are introduced in the $\mathrm{CdZnMgTe}$ barrier, well apart from the free carriers and the localized spins lying in the CdMnTe quantum well. With such a reduced disorder, it is possible to have a low density of free carriers, or, more precisely, a large number of $\mathrm{Mn}$ spins on a scale of the order of $k_{\mathrm{F}}^{-1}$ (which defines the typical length of the RKKY interaction). Typically, $k_{\mathrm{F}}^{-1}$ is of the order of $5-10 \mathrm{~nm}$ in the present quantum wells, compared to $0.7-5 \mathrm{~nm}$ in the case of the $\mathrm{ZnMnTe}$ layers and even less in GaMnAs. The difference in lengthscales is even greater if we consider the average $\mathrm{Mn}-\mathrm{Mn}$ distance projected in the plane of the quantum well. This is the relevant parameter since in the samples under study, only one subband is populated (with a typical kinetic energy at Fermi level about $4 \mathrm{meV}$ ). Hence a Mn spin, at 
a given position in the direction $z$ normal to the quantum well, could alter the envelope function along $z$ only through mixing with states at higher energy: Such mixing will be less efficient that those which give rise to the RKKY oscillations in a 3D system. If we neglect such mixing, we have to look whether an atomic column in the $z$ direction contains a $\mathrm{Mn}$ or not. Then in a 25 monolayer thick quantum well made of CdMnTe with $4 \% \mathrm{Mn}$, the probability to find at least one spin on a given atomic column is 0.64 . Note that this configuration is quite different from that of thin GaMnAs layers [17] where a large number of subbands is populated. Another difference is the fact that, in a thin GaMnAs layer, a strong disorder is expected from the presence of acceptors within the "quantum well".

The ferromagnetic interaction has been studied by photoluminescence spectroscopy, using the well known selection rules (the photoluminescence line observed in $\sigma^{+}$polarization involves the $|+3 / 2\rangle$ hole and the $\sigma^{-}$line - the $|-3 / 2\rangle$ hole) and the fact that the giant Zeeman splitting is proportional to the Mn magnetization. We observe a ferromagnetic enhancement of the susceptibility (i.e., of the slope of the Zeeman splitting vs. field), which diverges at a positive temperature $T_{\mathrm{CW}}$, and a zero-field splitting at a low temperature which is attributed to the spontaneous magnetization [5, 22]. The detailed calculation within the mean field model has been given elsewhere [14]. Here again the role of the structure of the valence band is important: We are dealing with heavy holes confined in the quantum well, which have (if we neglect heavy-hole light-hole mixing) no spin component in the directions within the quantum well. Thus Eq. (1) reduces to

$$
-\beta S_{i}^{z} s^{z} \delta\left(\boldsymbol{r}-\boldsymbol{R}_{i}\right)
$$

and the coupling between localized spins is not described by a Heisenberg Hamiltonian $J_{i j} \boldsymbol{S}_{i} \boldsymbol{S}_{j}$ (as usual in the RKKY description) but by an Ising Hamiltonian $J_{i j} S_{i}^{z} S_{j}^{z}$.

The photoluminescence lines remain sharp, so that the Zeeman splitting is well resolved even at a very low applied field. It is then particularly interesting to note the behaviour as the applied field is increased (Fig. 4). One observes a strong enhancement only at a very low field, and the normal giant Zeeman effect at a higher field. The low field behaviour corresponds to the ferromagnetic enhancement of the Mn susceptibility, induced by the interaction with the carriers. As soon as the carrier gas is fully polarized however, the magnetization of Mn spins is determined by a constant exchange field induced by the polarized carriers. Hence one recovers the usual giant Zeeman effect, shifted in field by the exchange field of the order of $1000 \mathrm{Oe}$. This spectroscopic observation rules out any separation into separate zones where the localized spins would be ferromagnetically coupled, or not, respectively, due to an inhomogeneous distribution of carriers, as suggested in the case of thick layers [3]: The observed photoluminescence is due to charged excitons [23], which are sensitive to the electrostatic potential fluctuations [24], as the carriers are, so that localization of carriers and localization of charged excitons are expected to exhibit similar length scales. Hence modulation doped 


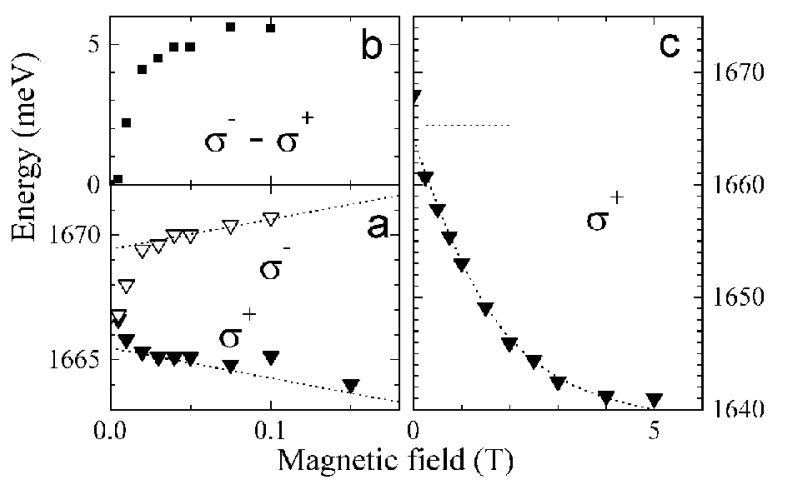

Fig. 4. Photoluminescence from a $\mathrm{Cd}_{0.97} \mathrm{Mn}_{0.03} \mathrm{Te} \mathrm{QW}$ with $1.6 \times 10^{11}$ holes $\mathrm{cm}^{-2}$; (a) transition energy in $\sigma^{+}$and $\sigma^{-}$polarizations at a low field; (b) energy differences from (a); (c) transition energy in $\sigma^{+}$and $\sigma^{-}$polarizations at a high field, and fit with a Brillouin function.

$\mathrm{Cd}_{1-x} \mathrm{Mn}_{x}$ Te quantum wells allow the observation of the ferromagnetic interaction induced by a homogeneous carrier gas with a very low density.

\section{Conclusion}

II-VI diluted magnetic semiconductors appear as model materials for the study of carrier induced ferromagnetic interactions in semiconductors. They allow a separate control of the introduction of carriers (doping with nitrogen) and localized spins (Mn), so that a large number of configurations can be achieved, including $3 \mathrm{D}$ thick layers and $2 \mathrm{D}$ modulation doped quantum wells. In the case of thick layers, disorder does not affect significantly the critical temperature, but appears to reduce the magnetization. In the quantum wells, a very low density of the carrier gas can be achieved with a homogeneous behaviour of the interacting spin + carrier system.

\section{References}

[1] P. Lazarczyk, T. Story, M. Arciszewska, R.R. Gałązka, J. Magn. Magn. Mater. 169, 151 (1997) and references therein.

[2] H. Munekata, H. Ohno, S. von Molnar, A. Segmuler, L.L. Chang, L. Esaki, Phys. Rev. Lett. 63, 1849 (1989).

[3] H. Ohno, Science 281, 951 (1998) and references therein.

[4] H. Ohno, D. Chiba, F. Matsukura, T. Omiya, E. Abe, T. Dietl, Y. Ohno, K. Ohtani, Nature 408, 944 (2000).

[5] A. Haury, A. Wasiela, A. Arnoult, J. Cibert, S. Tatarenko, T. Dietl, Y. Merle d'Aubigné, Phys. Rev. Lett. 79, 511 (1997).

[6] D. Ferrand, J. Cibert, A. Wasiela, C. Bourgognon, S. Tatarenko, G. Fishman, T. Andrearczyk, J. Jaroszynski, S. Kolesnik, T. Dietl, B. Barbara, D. Dufeu, Phys. Rev. B 63, 85201 (2001). 
[7] L. Hansen, D. Ferrand, G. Müller, V. Hock, N. Schwarz, G. Schmidt, A. Waag, MRS Fall Meeting, Boston 2000, umpublished.

[8] T. Dietl, H. Ohno, F. Matsukura, J. Cibert, D. Ferrand, Science 287, 1019 (2000).

[9] K. Sato, H. Katayama-Yoshida, Jpn. J. Appl. Phys. 39, 555 (2000).

[10] E. Kulatov, H. Nakayama, H. Mariette, H. Ohta, Yu.A. Upenskii, unpublished.

[11] K. Ueda, H. Tabata, T. Kawai, unpublished.

[12] Y. Matsumoto, M. Murakami, T. Shono, T. Hasegawa, T. Fukumura, M. Kawasaki, P. Ahmet, T. Chikyow, S. Koshihara, H. Koinuma, Science 291, 854 (2001).

[13] G.A. Medvedkin, T. Ishibashi, T. Nishi, K. Hayata, Y. Hasegawa, K. Sato, Jpn. J. Appl. Phys. 39, L949 (2000).

[14] T. Dietl, A. Haury, Y. Merle d'Aubigné, Phys. Rev. B 55, R3347 (1997).

[15] J. Schliemann, J. Konig, H.-H. Lin, A.H. MacDonald, Appl. Phys. Lett. 78, 1550 (2001).

[16] R.N. Bhatt, Mona Berciu, http://arXiv.org/abs/cond-mat/0011319.

[17] M.A. Boselli, A. Ghazali, I.C. da Cunha Lima, Phys. Rev. B 62, 8895 (2000).

[18] J.A. Gaj, R. Planel, G. Fishman, Solid State Commun. 29, 435 (1979).

[19] J.K. Furdyna, J. Appl. Phys. 64, R29 (1988); T. Dietl, in: Handbook on Semiconductors, Ed. T.S. Moss, Vol. 3b, North-Holland, Amsterdam 1994, p. 1251.

[20] T. Andrearczyk, J. Jaroszynski, M. Sawicki, Le Van Khoi, T. Dietl, D. Ferrand, C. Bourgognon, J. Cibert, S. Tatarenko, T. Fukumura, Zhengwu Jin, H. Koinuma, M. Kawasaki, in: Proc. 25th Int. Conf. Phys. Semiconductors, Osaka 2000, Eds. N. Miura, T. Ando, in series Springer Proc. in Physics, Vol. 87, Springer, Berlin 2001, p. 235.

[21] M. Grün, A. Haury, J. Cibert, A. Wasiela, J. Appl. Phys. 79, 7386 (1996).

[22] P. Kossacki, D. Ferrand, A. Arnoult, J. Cibert, S. Tatarenko, A. Wasiela, Y. Merle d'Aubigné, J.-L. Staeheli, J.-D. Ganiere, W. Bardyszewski, K. Swiatek, M. Sawicki, J. Wróbel, T. Dietl, in: EP2DS XIII, Ottawa 1999, Physica E 6, 709 (2000).

[23] J. Cibert, D. Ferrand, P. Kossacki, A. Arnoult, A. Wasiela, S. Tatarenko, M. Potemski, T. Dietl, in: NATO Advanced Research Workshop on Optical Probing of Many-Body Effects in Nanostructures, Würzburg (Germany) 2000, unpublished; J. Cibert, S. Tatarenko, D. Ferrand, A. Wasiela, T. Dietl, MRS Fall Meeting, Boston 2000, unpublished.

[24] D. Brinkmann, J. Kudrna, P. Gilliot, B. Hönerlage, A. Arnoult, J. Cibert, S. Tatarenko, Phys. Rev. B 60, 4474 (1999). 